\title{
A Hybrid Approach for Data Duplication and Network Coding
}

\author{
Jakob Belschner*, and Diomidis S. Michalopoulos ${ }^{\dagger}$ \\ *Deutsche Telekom AG, Strategy \& Technology Innovation, Darmstadt, Germany. Email: jakob.belschner@ telekom.de \\ ${ }^{\dagger}$ Nokia Bell Labs, Munich, Germany. Email diomidis.michalopoulos@nokia-bell-labs.com
}

\begin{abstract}
The extreme requirements for 5G ultra-reliable services call for new approaches for achieving a reliable operation of the Radio Access Network (RAN). Data Duplication as well as Network Coding in combination with multi-connectivity are corresponding approaches that promise increased RAN reliability. In this work, the advantages and disadvantages of both Network Coding and Data Duplication are studied. Moreover, a novel hybrid approach is proposed, which combines the advantages of both approaches. The effectiveness of the proposed hybrid approach is corroborated by means of simulations.
\end{abstract}

\section{INTRODUCTION}

The most common $5 \mathrm{G}$ services are extreme mobile broadband (eMBB), massive machine type communication (mMTC) and ultra-reliable low latency communication (URLLC) [1]. Such services differ in their nature and requirements. With respect to reliability, URLLC services are most challenging since the conventional methods used for increasing the probability of a successful reception in legacy systems become insufficient. Specifically, the extremely strict requirements on reliability reach the level of $99,999 \%$ probability of uninterrupted operation [2].

\section{Radio Access Network (RAN) Reliability}

In order to attain this extremely high level of reliability, alternative approaches are put forward for the RAN, such as Data Duplication (DD) and Network Coding (NC) [3][5]. DD achieves an increased reliability by duplicating data and sending it via two independent links (exploiting multiconnectivity). NC introduces an additional degree of freedom by generating multiple linear combinations out of a group of packets. NC can be used to increase the reliability by sending an additional amount of linear combinations to increase the decoding probability [6].

An illustrative view of the application of DD and $\mathrm{NC}$ in a multi-connectivity scenario is provided in Fig. 1. in particular, in the case of DD (left side of the Fig. 1), incoming packets are duplicated within a RAN reliability Virtual Network Function (VNF) in the telco cloud. Duplicates of the packets are forwarded towards the Mobile Station (MS) via two Distributed Units (DUs). A loss of one duplicated packet can be compensated by a successful reception of the same packet through the second link.

On the other hand, $\mathrm{NC}$ can be used as shown on the right side of Fig. 1. In this case, two (or more) incoming packets are combined. A set of different linear combinations (four in

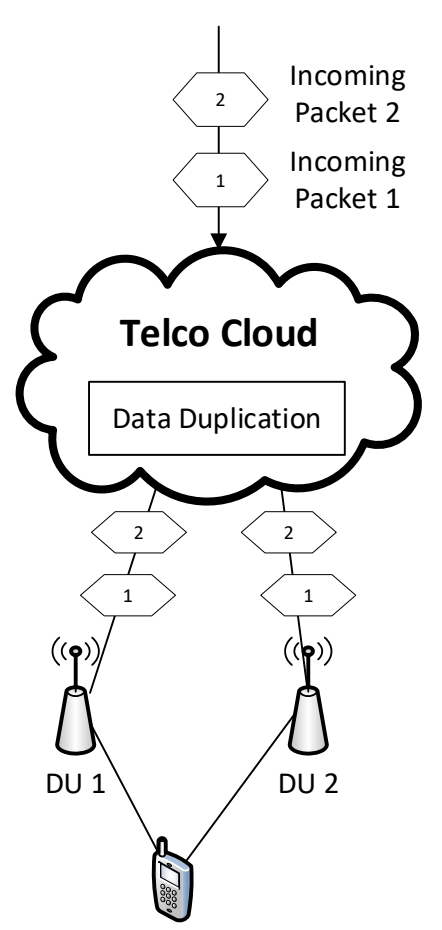

Mobile Station

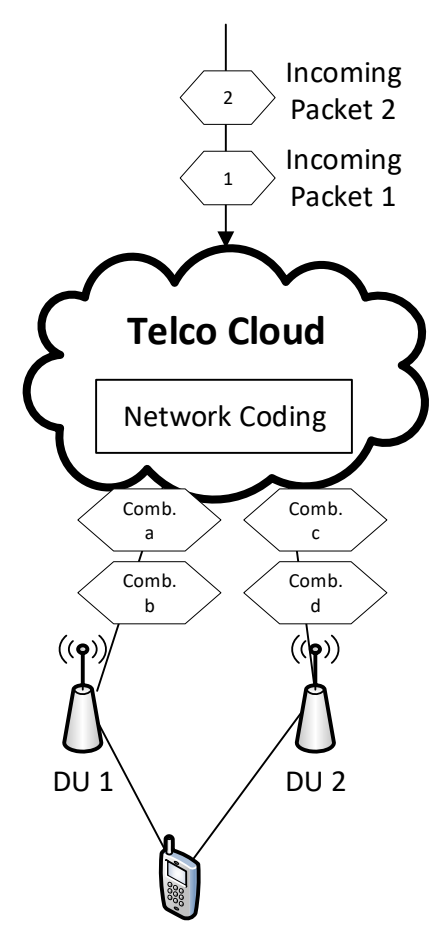

Mobile Station
Fig. 1: Improving RAN reliability by multi-connectivity in combination with Data Duplication (left side) and Network Coding (right side).

case of Fig. 1) is generated and sent via the two links. Even if two out of the four linear combinations are not successfully received, there is a high probability that both packets can be decoded at the MS [6].

\section{Contribution and Structure}

This work capitalizes on the use of DD and NC as effective RAN reliability approaches, and proposes a hybrid scheme for RAN reliability. The proposed hybrid scheme makes use of the advantages of both DD and $\mathrm{NC}$, in the sense that it can switch between the two techniques depending on the given requirements on reliability and latency. In this regard, a simulation framework is utilized for assessing the performance of the hybrid scheme. By means of such simulation framework, the conditions under which it is preferable to use DD over NC and vice versa are identified.

The proposed hybrid approach is introduced in the ensuing Section II, and then evaluated by means of simulations. The 
simulation methodology is described in Section III, whereas the obtained results are discussed in Section IV. The final concluding remarks are provided in Section $\mathrm{V}$.

\section{THE HYBRID APPROACH}

From a direct comparison analysis of DD and NC, one can realize that there are advantages and disadvantages for both of them, which are listed below.

- NC has the potential to achieve a higher reliability compared to DD. Taking the example of Fig. 1, NC could compensate the loss of combinations $a$ and $b$, if combinations $c$ and $d$ are received (or in general any two combinations). In the case of DD, the same event (the loss of both duplicates of packet one) would lead to a packet loss.

- DD has advantages in terms of latency: Packets that arrive at the DD VNF can be processed immediately. In the case of NC, a first packet might have to be queued to combine it with a second or third packet.

Combining the advantages of both schemes is the motivation for creating a hybrid approach. This hybrid approach is assumed to reside in the Telco Cloud as a VNF and flexibly adjust the RAN reliability strategy by switching between NC and DD. This switching between the two techniques takes place based on the following rules:

1) If multiple packets arrive at the RAN reliability VNF simultaneously, they are combined using NC to exploit the previously mentioned reliability gain. This occurs based on the NC generation size, which is fixed value. It determines the number of packets which form one generation out of which the linear combinations are derived. If e.g. four packets arrive and the generation size is set to two, packets one and two are combined as well as packets three and four.

2) If there are remaining packets which were not combined with other packets (e.g. packet five in the case of five packets arriving and a generation size of two), they are queued for a short time according to a configuration parameter. If no further packets arrive within this duration, the queued packets undergo DD and are sent out.

3) If a single packet arrives, it undergoes the same procedure described under point 2 .

\section{Simulation Methodology}

A simulation was executed to evaluate the performance of the hybrid approach compared to DD and NC. Fig. 2 shows the simulation setup. A traffic generator creates packets and forwards them to the VNF for RAN reliability. The VNF generates coded or duplicated packets according to the selected scheme (DD, NC, hybrid). The coded / duplicated packets are then sent via two links. For each link, assumptions on its performance (reliability versus delay) are made; such assumptions are introduced below. It is also possible to correlate the behaviour of both links, i.e. to increase the probability of simultaneous errors. Both links terminate at the same decoder which reconstructs the original packets.

The performance of the underlying links heavily influences the resulting reliability at the decoder. To study this, in the following three different air interfaces are studied. Their performance is depicted in Fig. 3. The blue curve represents an Ultra-Reliable Low-Latency Communication (URLLC) air interface [2]. This air interface is able to deliver packets after $0.3 \mathrm{~ms}$ with an error rate of $10^{-2}$. After $1 \mathrm{~ms}$, packets can be delivered with an error rate of $10^{-5}$. The yellow curve represents the behaviour of LTE with $1 \mathrm{~ms}$ latency for an error rate of $10^{-1}$. It should be noted that the performance of an URLLC air interface can only be achieved under the constraint of a significantly lower spectral efficiency [7], [8]. Therefore, a third air interface is additionally assumed (red curve), which is targeted to achieving a compromise between reliability and spectral efficiency. To achieve high spectral efficiency, the third air interface targets a relatively low reliability $\left(10^{-1}\right.$ error rate) and uses a $1 \mathrm{~ms}$ Time Transmission Interval (TTI), but at the same time performs retransmission more quickly than LTE. With a corresponding parameterization, this performance should be achievable with $5 \mathrm{G}$ NR.

Besides the lower layer performance, also the traffic model, i.e. the timing of the incoming packets, has a significant impact on the performance of NC and the hybrid approach. Two traffic models have been simulated:

- A traffic model with uniform inter-arrival time of the packets. In this case, a packet is followed by the next one after a fixed time. This traffic model represents industrial fieldbus traffic [9]. To create a best case scenario for NC, a large number of packets (10,000 packets per second) was assumed here, such that the queuing time for combining one packet with another is low.

- In contrast, a bursty traffic model was assumed, which represents e.g., file transfers. In this case, bursts of in average 50 packets (with a standard deviation of 4 packets) were generated. One burst spans over $1 \mathrm{~ms}$ and in average 500 bursts per second are generated.

\section{Simulation Results}

In the following, simulation results for the three different lower layer performances and the two traffic types are presented. After that also simulation results which study the impact of correlated links are provided.

Fig. 4 shows the performance for the case of the URRLC air interface and bursty traffic. A significant drawback of the NC approach can been seen: due to the queueing effect described in Section II, some packets are delayed, which influences the overall performance negatively. In this respect, one should note that, for instance, for a packet error rate of $10^{-4}$, one delayed packet out of 10,000 packets affects the overall performance. On the other hand, DD achieves the expected performance: as a single link achieves an error rate of $10^{-2}$ after $0.3 \mathrm{~ms}$ (blue curve in Figure 3 ) it achieves an error rate of $10^{-4}$ after $0.3 \mathrm{~ms}$ by exploiting two uncorrelated multiconnectivity links in Fig. 4. The hybrid approach achieves a significant reliability increase compared to DD by combining most of the packets via NC while simultaneously avoiding the queuing problems of NC. For the hybrid approach, as well as $\mathrm{NC}$, different generation sizes, i.e., the number of packets that were combined in one group, were simulated. This had low impact for the simulated conditions.

Fig. 5 shows the performance for the case of the URRLC air interface and uniform traffic. The scaling of the $\mathrm{x}$-axis is 


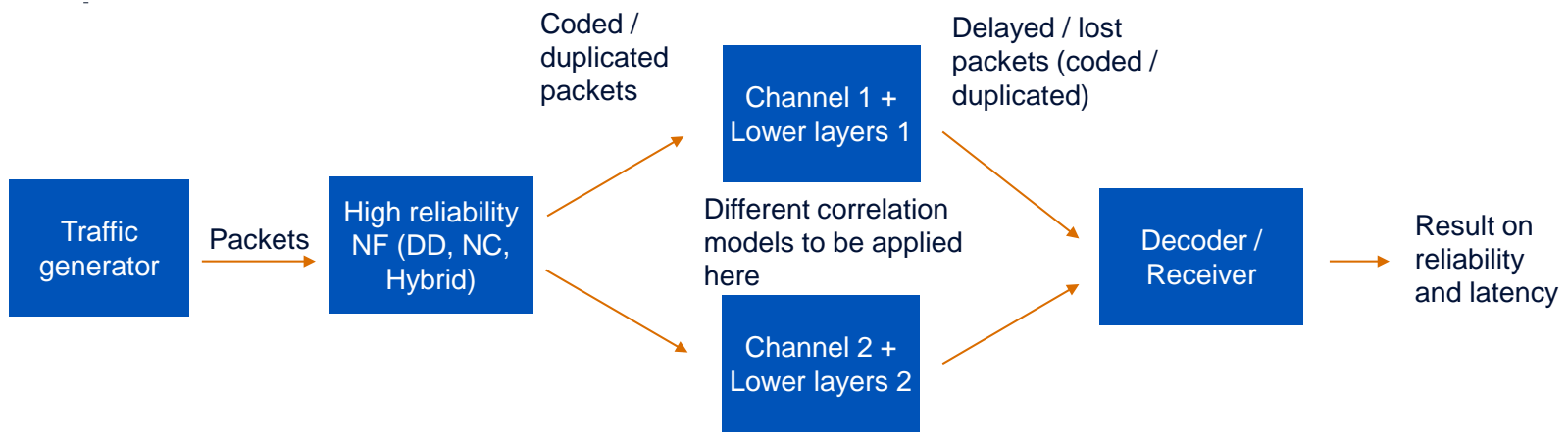

Fig. 2: Simulation setup for analyzing Network Coding, Data Duplication and the hybrid approach

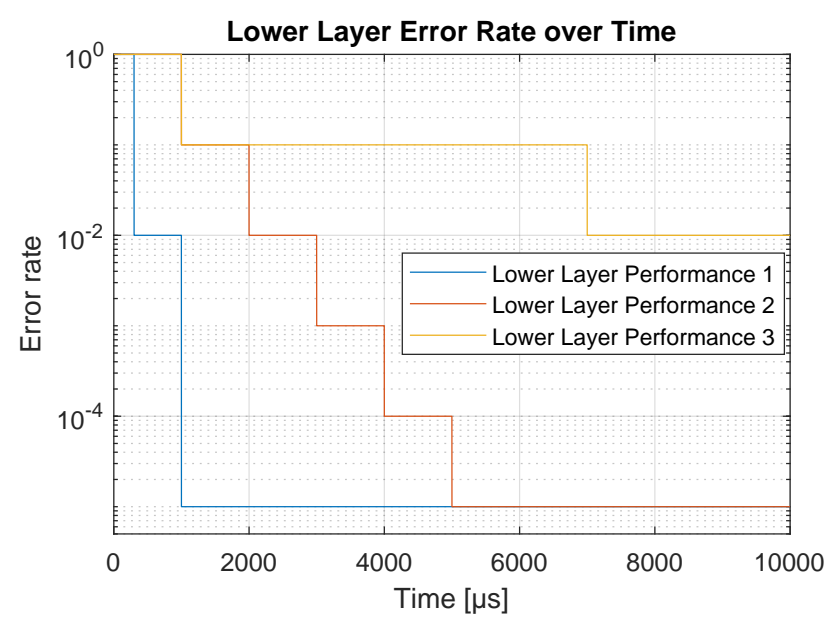

Fig. 3: Simulation Assumption: Lower Layer Performance

changed compared to that in Fig. 4 to allow for more insights on the performance at low latency. NC and the hybrid approach in this case achieve the same performance, such that the green and the blue curve coincide (NC generation size 2 achieves the same performance as the hybrid approach with generation size 2; NC generation size 3 achieves the same performance as the hybrid approach with generation size 3 and so on).

Uniform traffic with a high packet rate is the best case for NC, since a low queuing delay is required until a second or third packet arrives. Therefore NC achieves a significant increase in reliability, with a low penalty in terms of latency. Higher generation sizes lead to higher reliability on the cost of latency. The hybrid approach in this case was configured via the configuration parameter (described in Section II) such that it queues the packets until one $\mathrm{NC}$ generation can be created. It therefore achieves the same performance as NC.

Figs. 6 and 7 show the simulation results for the air interface with reduced reliability (red curve in Fig. 3). The same trends as observed for the URLLC air interface can been seen here:

- NC has a significant drawback in the case of bursty traffic.

- The hybrid approach achieves the best performance in the bursty traffic case, in the case of uniform traffic it

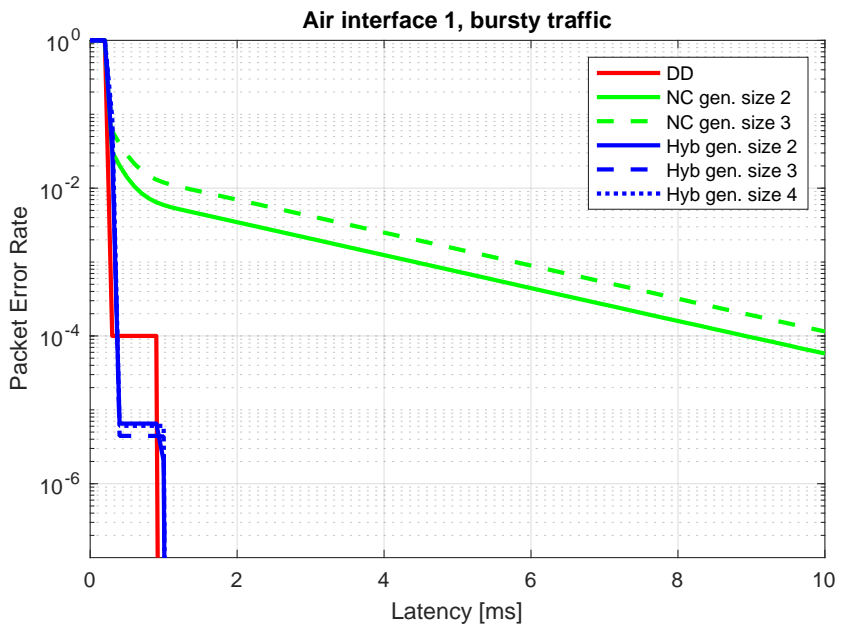

Fig. 4: Simulation results for bursty traffic and URLLC air interface

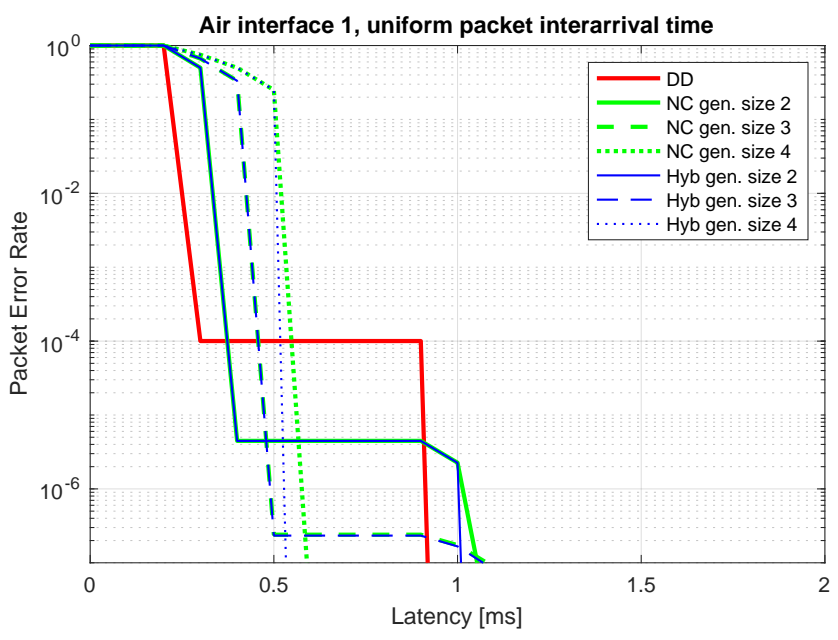

Fig. 5: Simulation results for uniform traffic and URLLC air interface 


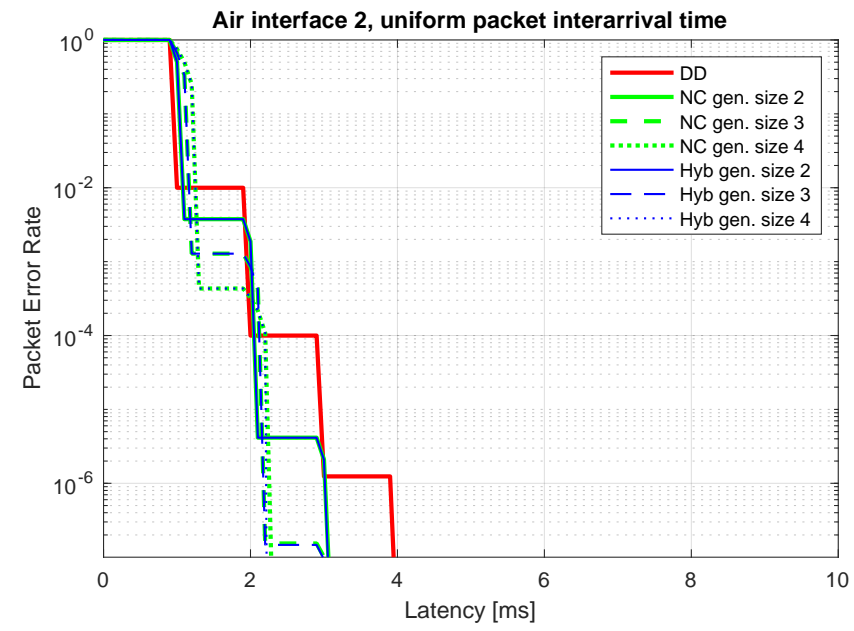

Fig. 7: Simulation results for uniform traffic and medium air interface

achieves the performance of $\mathrm{NC}$ (the green curves are again coinciding with the blue ones).

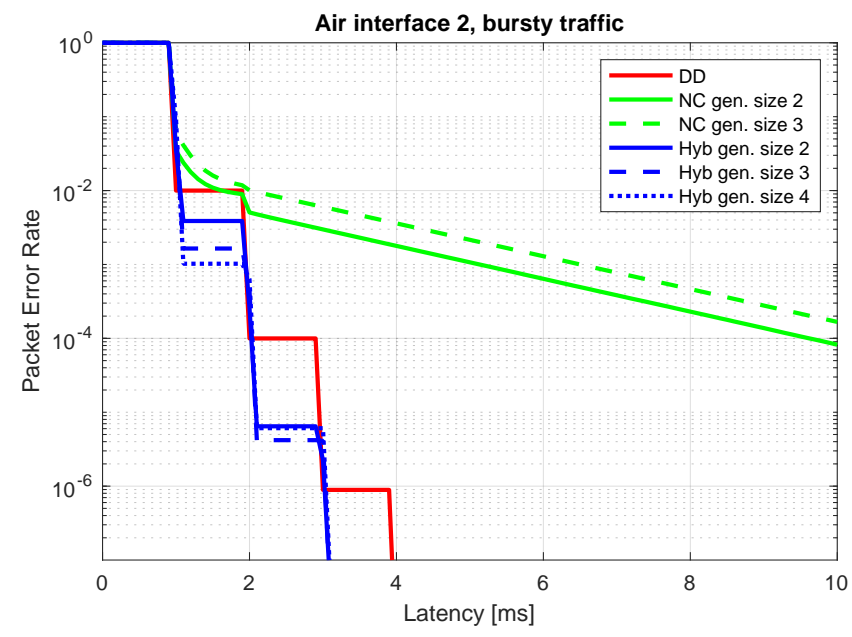

Fig. 6: Simulation results for bursty traffic and medium air interface

Figs. 8 and 9 show the simulation results for the air interface with low reliability (yellow curve in Fig. 3). The following trends can be seen:

- Due to the lower overall reliability, NC can compensate the drawbacks in case of bursty traffic and achieves a performance similar to DD.

- For the uniform traffic the hybrid approach again achieves the same performance as NC. Due to combining packets and multi-connectivity, this leads to the fact that even with the relatively unreliable air interface, a packet error rate of $10^{-5}$ or less can be achieved if a higher latency is tolerated.

The results shown so far assumed two uncorrelated links towards the MS, which is a best-case assumption. In the

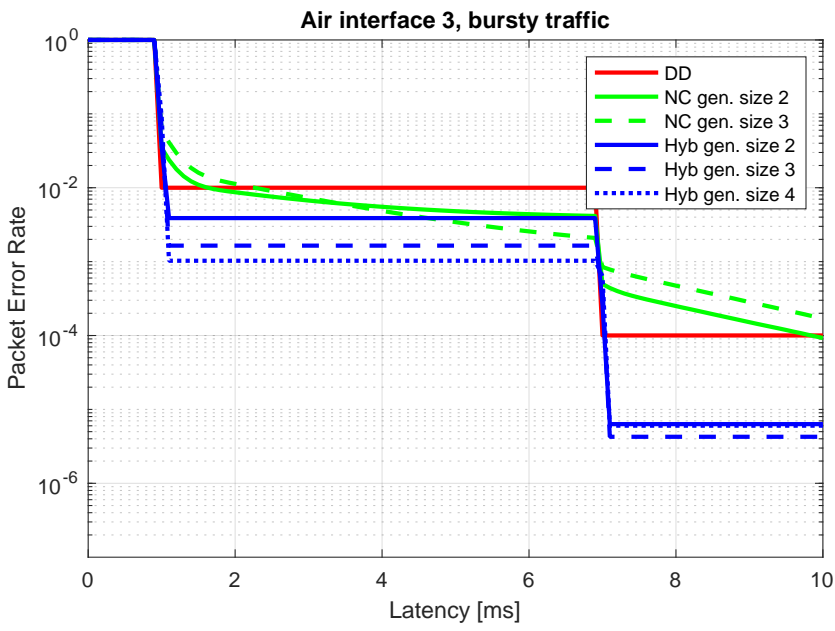

Fig. 8: Simulation results for bursty traffic and air interface with low reliability

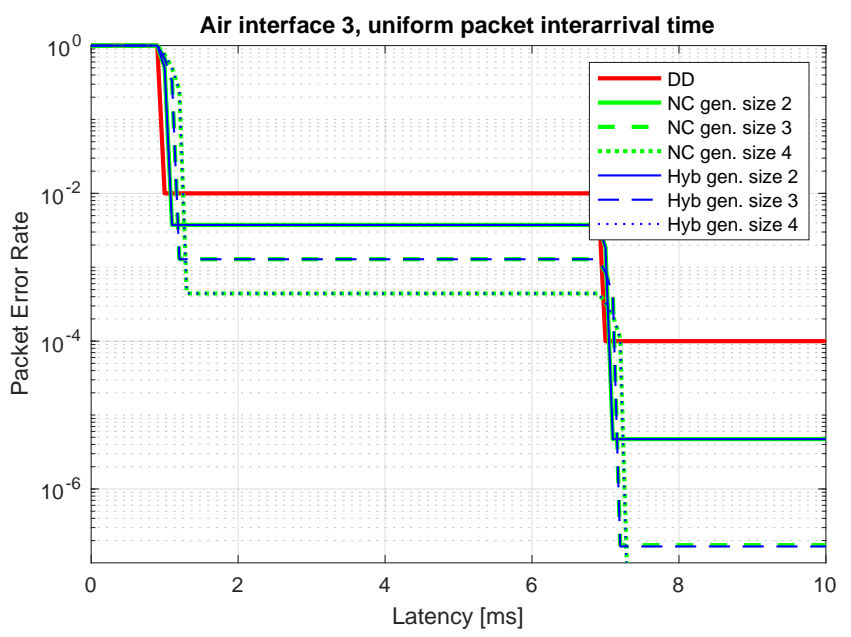

Fig. 9: Simulation results for uniform traffic and air interface with low reliability

following, the impact of correlated links is studied for the example of the air interface with interface of medium reliability. For this purpose, a correlation factor is introduced:

- A correlation factor of 0 means that both links cause independent packet losses.

- A correlation factor of 0.5 means that half of the errors occur simultaneously at both links, whereas the other half occurs uncorrelated.

- A correlation factor of 1 means that only simultaneous errors on both links occur.

Figs. 10 and 11 illustrate the corresponding results. It can be seen that, as expected, correlated links have a strong negative impact on reliability. In the extreme case of fully correlated links, DD is not a suitable means for increasing reliability and achieves the same performance as a single link. NC and the hybrid approach can compensate also for simultaneous errors (as introduced in Section II) but on the 


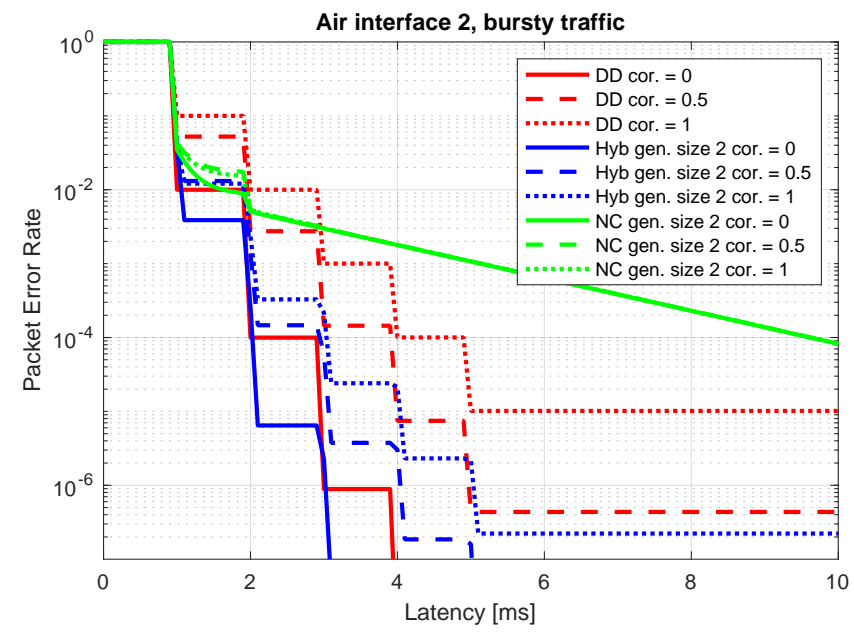

Fig. 10: Simulation results for correlated links and bursty traffic

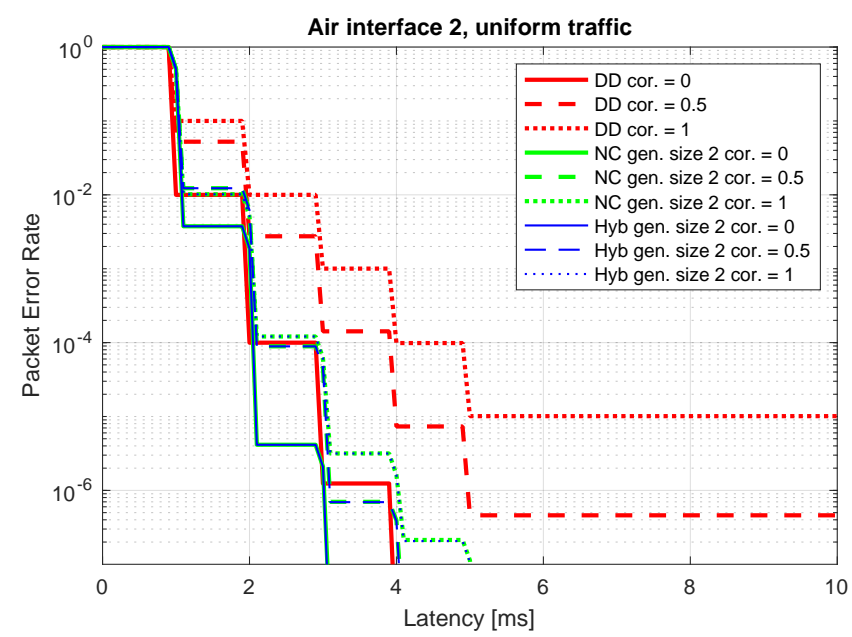

Fig. 11: Simulation results for correlated links and uniform traffic

expense of the reliability they achieve.

\section{CONCLUSion}

The work proposed and assessed a hybrid approach for Data Duplication and Network Coding. Simulation results demonstrated that the proposed hybrid approach can combine the advantages of both schemes. It furthermore achieves the highest reliability in the case of bursty traffic, as well as equal performance compared to Network Coding in the case of uniform traffic. It is also shown that by combining packets, as in the case of Network Coding and the hybrid approach, the negative impact of correlation in the case of multi-connectivity can be significantly reduced compared to Data Duplication.

\section{ACKNOWLEDGMENT}

This work has been performed in the framework of the H2020-ICT-2016-2 project 5G-MoNArch. The authors would like to acknowledge the contributions of their colleagues. This information reflects the view of the consortium, but the consortium is not liable for any use that may be made of any of the information contained therein.

\section{REFERENCES}

[1] S. E. Elayoubi, M. Fallgren, P. Spapis, G. Zimmermann, D. MartinSacristan, C. Yang, S. Jeux, P. Agyapong, L. Campoy, Y. Qi, and S. Singh, "5G service requirements and operational use cases: Analysis and METIS II vision," in 2016 European Conference on Networks and Communications (EuCNC). IEEE, jun 2016, pp. 158-162. [Online]. Available: http://ieeexplore.ieee.org/document/7561024/

[2] G. Pocovi, K. I. Pedersen, and P. Mogensen, "Joint Link Adaptation and Scheduling for 5G Ultra-Reliable Low-Latency Communications," IEEE Access, vol. 6, pp. 28912-28922, 2018. [Online]. Available: https://ieeexplore.iee.org/document/8361404/

[3] J. Rao and S. Vrzic, "Packet duplication for urllc in 5g dual connectivity architecture," in 2018 IEEE Wireless Communications and Networking Conference (WCNC), April 2018, pp. 1-6.

[4] P. Wang, G. Mao, Z. Lin, and X. Ge, "An efficient network coding based broadcast scheme with reliability guarantee," in 2014 IEEE International Conference on Communications (ICC), June 2014, pp. 2873-2878.

[5] H2020 ICT 2016 project 5G-MoNArch, "Deliverable D3.1: Initial resilience and security analysis," Jun 2018.

[6] O. Trullols-Cruces, J. M. Barcelo-Ordinas, and M. Fiore, "Exact decoding probability under random linear network coding," IEEE Communications Letters, vol. 15, no. 1, pp. 67-69, 2011.

[7] Next Generation Mobile Networks Alliance, "5G Extreme Requirements: Radio Access Network Solutions 5G Extreme Requirements," Tech. Rep., 2018. [Online]. Available: https://www.ngmn.org/publications/technicaldeliverables.html

[8] J. Sachs, G. Wikstrom, T. Dudda, R. Baldemair, and K. Kittichokechai, "5G Radio Network Design for Ultra-Reliable Low-Latency Communication," IEEE Network, vol. 32, no. 2, pp. 24-31, mar 2018. [Online]. Available: http://ieeexplore.ieee.org/document/8329620/

[9] A. Willig, K. Matheus, and A. Wolisz, "Wireless Technology in Industrial Networks," Proceedings of the IEEE, vol. 93, no. 6, pp. 1130-1151, jun 2005. [Online]. Available: http://ieeexplore.iee.org/document/1435743/ 